\title{
Mechanical Design of the LSST Camera
}

\author{
Martin Nordby ${ }^{*}$, Gordon Bowden ${ }^{\mathrm{a}}$, Mike Foss ${ }^{\mathrm{a}}$, Gary Guiffre ${ }^{\mathrm{a}}$, John Ku ${ }^{\mathrm{b}}$, Rafe Schindler ${ }^{\mathrm{a}}$ \\ ${ }^{\mathrm{a}}$ Stanford Linear Accelerator Center ${ }^{\dagger}, 2575$ Sand Hill Rd, Menlo Park, CA 94025 \\ ${ }^{\mathrm{b} S t e l l a r}$ Solutions, Inc., 250 Cambridge Ave Ste 204, Palo Alto, CA 94306
}

\begin{abstract}
The LSST camera is a tightly packaged, hermetically-sealed system that is cantilevered into the main beam of the LSST telescope. It is comprised of three refractive lenses, on-board storage for five large filters, a high-precision shutter, and a cryostat that houses the 3.2 giga-pixel CCD focal plane along with its support electronics. The physically large optics and focal plane demand large structural elements to support them, but the overall size of the camera and its components must be minimized to reduce impact on the image stability. Also, focal plane and optics motions must be minimized to reduce systematic errors in image reconstruction. Design and analysis for the camera body and cryostat will be detailed.
\end{abstract}

Keywords: LSST, camera, mechanical, design, structure, stable, mechanism, vacuum, thermal

\section{INTRODUCTION}

The Large Synoptic Survey Telescope (LSST) camera lies at the core of the telescope. The 3.2 giga-pixel CCD focal plane forms the heart of the instrument, while the camera is cantilevered into the center of the three-mirror telescope.

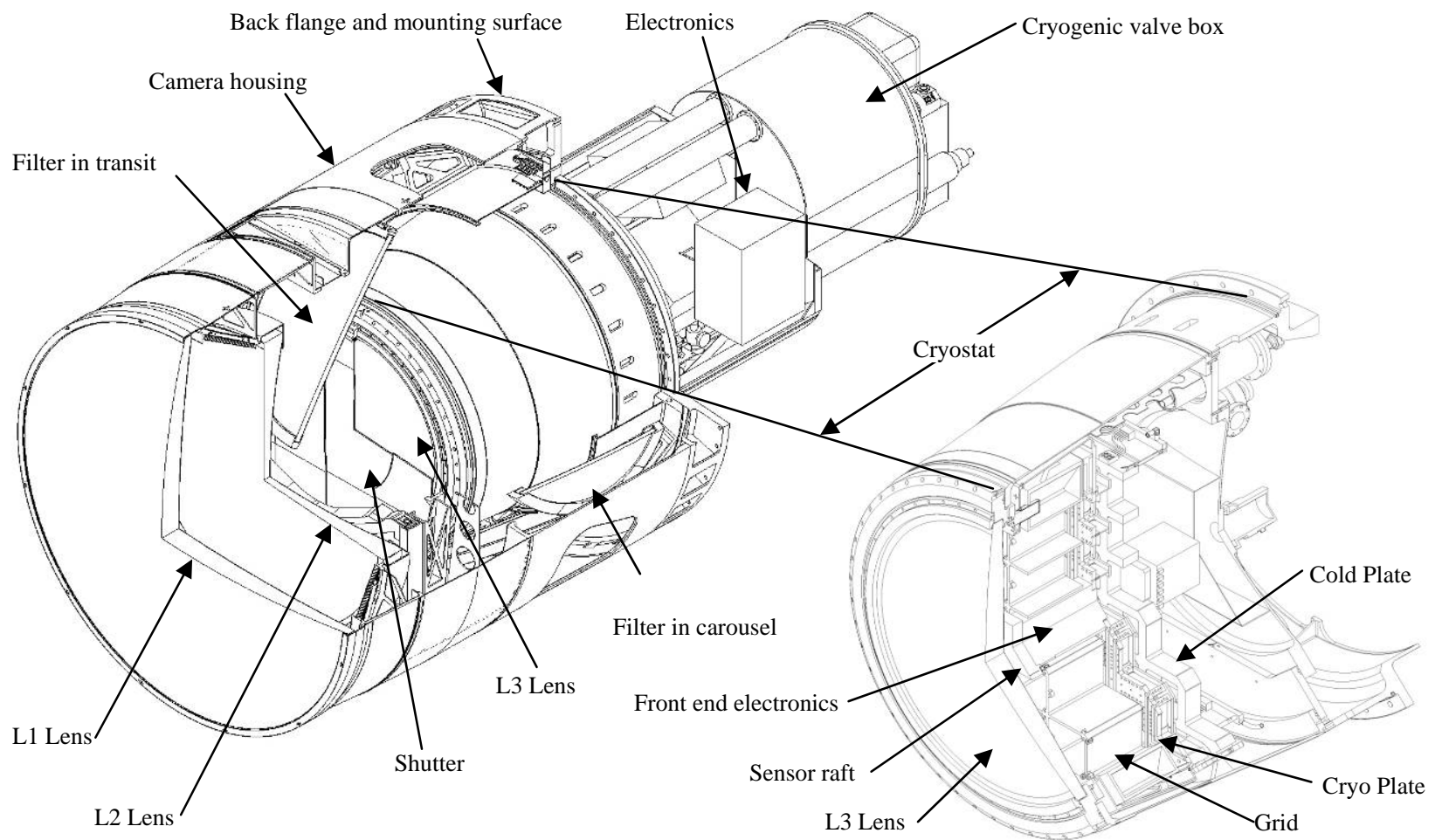

Fig. 1. Section of the LSST Camera, left, and an enlarged section of the cryostat and its contents

\footnotetext{
*nordby@slac.stanford.edu; phone 1-650-926-3415; www.slac.stanford.edu

$\dagger$ Work supported by the Department of Energy contract DE-AC02-76SF00515
} 
As shown in Fig. 1, the camera is a fully-enclosed, self-contained assembly, composed of functional sub-assemblies that themselves are modular. The cryostat lies in the center of the camera. Its vacuum housing supports the focal plane of CCD detectors that operate at $-100{ }^{\circ} \mathrm{C}$, as well as the support and read-out electronics. Structural support and thermal control systems maintain suitable operating environments and isolate the focal plane from external dynamic and transient loads.

The camera includes four optical elements: the large L1 and L2 refractive lenses at the entrance to the camera, followed by a filter and finally the L3 lens, the window into the cryostat. Five filters are stored on-board the camera, allowing for quick filter changes during observing runs. The shutter lies between the filter and L3 lens. Support electronics and cryogenic services are mounted behind the camera in a utility volume that moves with the camera, while the outer housing supports the structural loads of the $3000 \mathrm{~kg}$ camera cantilevered off of the telescope's rotator.

The camera structural and thermal design responds to four classes of requirements. First, thermal and structural stability are essential to reduce systematic errors that would impact the performance of a survey telescope such as LSST. Second, particulates and contamination must be controlled to minimize image quality degradation. Third, because the camera sits between the telescope mirrors, it occludes the incoming light beams. Thus, its size must be reduced to the minimum needed for its optical elements. Fourth, given that LSST is a survey telescope, the mechanical systems must be reliable, with a high up-time.

High-level camera requirements and parameters are shown in Table 1, while more detailed requirements for camera subsystems are tabulated in their appropriate sections. In differing ways, the design of each camera subsystem is affected by one or more of these high-level requirements. In the following sections, each subsystem is discussed, along with its derived requirements and consequent design features, which result in a camera that is designed to perform as needed for a next-generation survey telescope such as LSST.

\section{CRYOSTAT STRUCTURAL SUPPORT SYSTEM}

\subsection{Design overview}

The cryostat is comprised of three systems of hardware: the structural, thermal, and contamination control systems, with the design of each system driven largely by requirements involving isolation and stability. We will review each hardware system, their driving requirements, and details of the design that respond to these requirements.

The cryostat structural support system includes the components that provide the support for the CCD sensor rafts in the cryostat vacuum. These components include the cryostat housing, grid structure and support flexures, and the kinematic coupling interface to the sensor rafts. Fig. 2 shows an isolation of these components in the cryostat. The stainless steel cryostat housing is cantilevered off of the back flange of the camera. It is tapered to form a truncated cone, to better channel the gravity loads from the end of the cantilever back to the support flange, thereby reducing gravity-induced deflections. The Grid is supported off the front flange of the housing by three titanium flexures. The flexures structurally and thermally isolate the cold Grid from the warm housing, and provide a stable three-point interface for support of the Grid. The Grid itself individually supports the 21 sensor rafts, holding them in the focal plane by way of a three-point kinematic coupling.

\subsection{Driving requirements}

The single most significant requirement for the cryostat structural support system is the need to hold the CCD sensors flat on the focal plane to 10 microns. This requirement can only be met by structurally and thermally isolating the support structure from as many external disturbances as possible. Table 2 shows a more complete list of structural requirements for the cryostat components. 


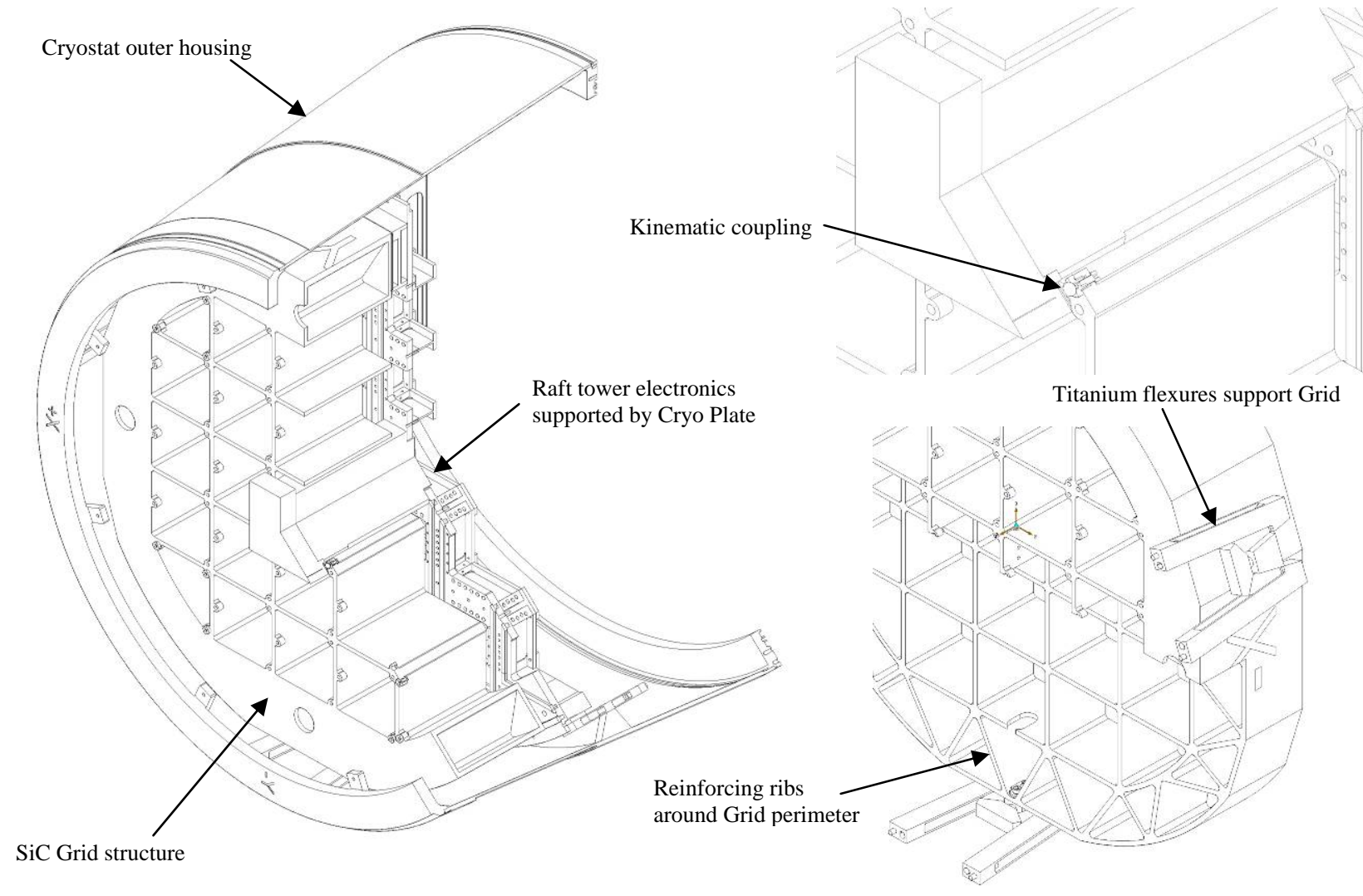

Fig. 2. Details of Cryostat structural elements with all other components not shown: the section view on the left shows the Grid with one of the twenty-one raft towers inserted, backed by the Cryo Plate; the upper right detail shows the kinematic ball interfaces between the sensor raft and the Grid, while the cut-away detail in the lower right shows the Grid perimeter reinforcement and two of the three support flexures.

\subsection{Kinematic coupling}

The first point of isolation lies at the interface between the sensor rafts and the Grid. This connection is accomplished by a kinematic coupling known as a Maxwell mount, more colloquially referred to as a three-vee mount. Here, the back side of the raft features three radially-arrayed vee's ground into the parent material, and used for aligning the CCD sensor packages. These vees position the raft to silicon-nitride ceramic balls that are fixed in cups mounted to the Grid. This forms a perfect six-point kinematic connection that is insensitive to grid distortion, assembly tolerances, and to the expected thermal motions across the joint due to differential contraction during cool-down..

\subsection{Grid design and materials}

Because the LSST camera pitches and rolls with the motion of the telescope, the flatness of the CCD's on the focal plane is dependent on the structural stiffness of the Grid to maintain planarity, despite the changing direction of gravitational loads. However, the Grid must also remain very open to allow for installation of the sensor rafts and their accompanying electronics. To allow for both an open central structure and an overall stiff design, the Grid incorporates a closed box-section ring that completely surrounds the central open region. The box structure is further reinforced with internal ribs to provide added shear and torsional stiffness.

Table 2. Focal plane requirements and parameters

\begin{tabular}{|l|l|}
\hline \multicolumn{1}{|c|}{ Property } & \multicolumn{1}{c|}{ Value } \\
\hline Clear field of view & $634 \mathrm{~mm}$ diameter \\
\hline Focal plane sensor flatness & $10 \mu \mathrm{m}$ peak-to-valley \\
\hline Pixel pitch & $10 \mu \mathrm{m}(0.2 \mathrm{a}-\mathrm{sec})$ \\
\hline Fill factor & $>90 \% \quad \begin{array}{l}126.5 \quad \mathrm{~mm} \text { square } \\
9 \times 42 \mathrm{~mm} \text { sq CCD's }\end{array}$ \\
\hline Sensor raft size & $94.5 \mathrm{~kg}$ \\
\hline Mass of focal plane ass'y
\end{tabular}


The Grid is manufactured from a silicon-carbide ceramic matrix composite. This material is used in the aerospace industry for lightweight and stable space-borne structures, including focal planes, optical benches, and mirrors. However, this will be the first known application of SiC for a ground-based instrument. The reason for the choice is clear when $\mathrm{SiC}$ material properties are compared with aluminum in Table 3. Both the specific modulus $(\mathrm{E} / \rho)$ and effective conductivity $(\mathrm{k} / \alpha)$ of $\mathrm{SiC}$ are superior to aluminum - a material commonly used for focal plane support structures on smaller cameras. Furthermore, the improved fracture toughness of the ECM CeSic ${ }^{\circledR}$ material makes it ideal for the Grid structure.

\subsection{Structural isolation and mass off-loading}

The final feature of the structural design that responds to the requirements of focal plane flatness and stability is structural isolation. This is partially accomplished by the flexures that support the Grid, which completely isolate it from loading due to external deformations. The flexures support the Grid in exactly six degrees of freedom, allowing us to fully characterize Grid distortions and count on repeatable gravity-induced deflections that will not be influenced by changes in the configuration of external structures.

Furthermore, the Grid supports as little mass as possible-only the sensor rafts and their supports-to reduce gravityinduced deflections. This has been accomplished by structurally decoupling each raft from its front-end support electronics. The electronics crate is supported by a neighboring cryogenically-cooled plate, the Cryo Plate, while the sensor rafts are held by the Grid. The rafts are held in position by springs that pre-load the rafts against their kinematic coupling, producing a uniform, invariant loading. While the inertial mass supported by the Grid has been reduced, the deflection due to the external spring loading is compensated for during initial integration, then remains unchanged, independent of camera orientation and temperature.

\section{CRYOSTAT THERMAL SYSTEM}

\subsection{Design overview}

As shown in Fig. 3, the primary components of the cryostat thermal system are the cryo plate and the cryo shroud. The cryo plate provides a heat sink for the sensor front end electronics and the CCD sensors themselves, as well as structurally supporting the electronics modules. It is mounted directly behind the Grid with thermally isolating flexures supported off the inside of the cryostat wall. The cryo shroud is mounted and thermally grounded to the perimeter of the cryo plate, and completely surrounds the Grid around its perimeter and front face. The shroud is cooled by the cryo plate, and supports multi-layer insulation (MLI) to provide a radiation barrier to the inside of the warm cryostat wall.

\subsection{Driving requirements}

Two key requirements drive the thermal design of the cryostat components; these and other parameters are listed in Table 4. First, the CCD sensors must be maintained at a stable operating temperature to within $0.3 \mathrm{~K}$. This is vital, since detector quantum efficiency varies strongly with temperature and must be stabilized to minimize systematic errors. Furthermore, this must be done despite changing radiative heat loading of the sensors across the focal plane, due to a radial temperature gradient in the L3 lens inner surface, directly across from the sensors.

A derived thermal requirement involves the need for temperature stability and uniformity within the Grid itself. Even small changes in the average Grid temperature or temperature gradients will thermally distort the Grid and move the sensors out of their required flatness on the focal plane.

\subsection{Parallel thermal paths}

The primary means by which the two driving thermal requirements are met is by establishing parallel thermal paths. Thermal straps remove the electrical and radiant heat load from each raft individually, and conduct it back to the cryo plate. Here, the conduction path ties in with parallel cross-flow cryogenic fluid circuits that minimize temperature gradients across the cryo plate. Separate cooling paths provide conductive cooling for the front end electronics, and 
thermal grounding for the Grid. Parallel cooling also allows for independent control of the temperature of each raft with low-wattage make-up heaters, to stabilize CCD temperatures over time. Such a small, local feedback loop is inherently more stable than a larger, more driven system that attempts to manage the overall temperature of the entire cryo plate.

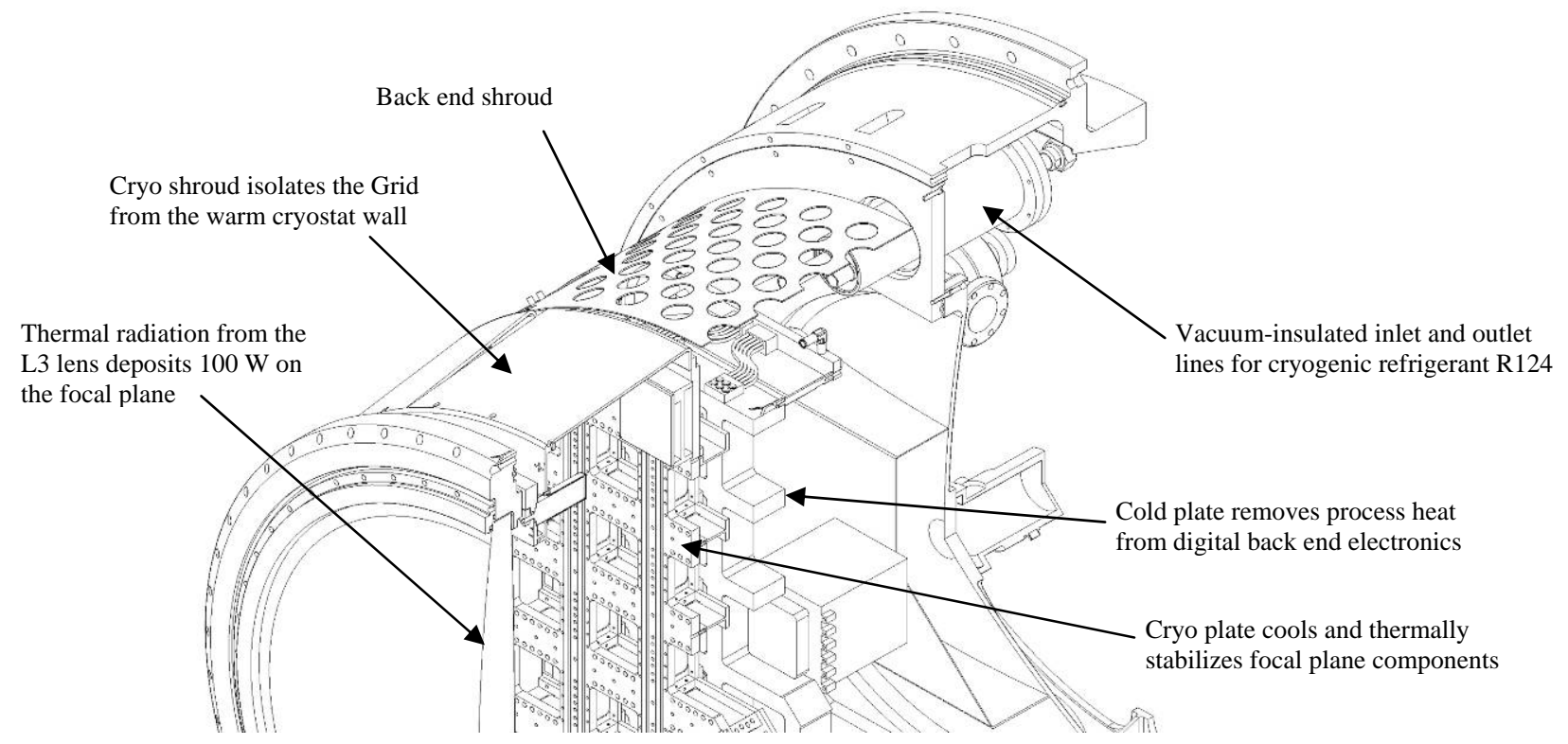

Fig. 3. Cryostat thermal components, with the outer housing and Grid support structure not shown. The cryo shroud surrounds the Grid to radiatively isolate it, while the cryo plate and cold plate provide thermal grounding for all cryostat components. The perforated radiation shroud isolates the back end of the cryostat from radiation heat leaks.

\subsection{Grid thermal isolation}

An important side benefit of such a parallel thermal design is that the Grid plays a purely passive role, thermally. The thermal design intentionally isolates the Grid from conducting any heat whatsoever, to avoid producing temperature gradients that could distort the Grid.

The thermal design goes one step further in radiatively isolating the Grid on all sides. In particular, the cryo shroud surrounds the Grid on its perimeter and front face. This provides a very uniform radiative environment for the Grid, since it will always be looking at the insulated cryo shroud, and not the room-temperature cryostat housing. This reduces the heat load on the Grid, and further isolates it from heat flow that could otherwise distort it.
Table 4. Thermal system key requirements and parameters

\begin{tabular}{|l|l|}
\hline \multicolumn{1}{|c|}{ Property } & \multicolumn{1}{c|}{ Value } \\
\hline Focal plane temperature & $173 \mathrm{~K}$ \\
\hline CCD sensor temperature stability & $0.3 \mathrm{~K}$ \\
\hline Radiative heat load through L3 & $100 \mathrm{~W}$ \\
\hline Grid temperature-induced distortion & $0.5 \mu \mathrm{m}$ \\
\hline Grid temperature uniformity & $0.5 \mathrm{~K}$ \\
\hline
\end{tabular}

\section{CRYOSTAT CONTAMINATION CONTROL}

\subsection{Design overview}

Due to the very large number of electronics channels, all of the front end analog, clock, and digitizing electronics reside inside the vacuum of the cryostat. This reduces driver voltages, minimizes cross-talk, and significantly reduces the pin count for feedthroughs out of the cryostat. However, this also introduces possible contamination sources into the cryostat that could outgas and coat the CCD detectors or fog the inside of the L3 lens.

\subsection{Key requirements}

The optical cleanliness of the CCD and L3 surfaces is of paramount concern. This translates into a requirement that all materials that are used in the cryostat must be qualified for use in our optical system. Further, any outgassing that does occur must be minimized, and the effects of the outgassing must be reduced as much as possible. 


\subsection{Cryostat thermal zones}

The cryostat design implements three general principles to reduce the risk of contamination. First, the cryostat has been segmented into four separate thermal zones, as depicted in the schematic in Fig. 4. The $-120{ }^{\circ} \mathrm{C}$ Cryo Plate and shroud serves as the coldest of the thermal zones, keeping all front end electronics cold to reduce outgassing and to provide cryo pumping. The CCD's on the focal plane form the second thermal zone. This is intentionally not the coldest zone, to reduce the cryo-pumping load on the CCD's. A $-40{ }^{\circ} \mathrm{C}$ cold plate forms the third thermal zone, structurally supporting and thermally grounding the power, clock, and digitizing electronics for each raft. Keeping these electronics cold reduces their gas load in the cryostat. The fourth thermal zone is the warm cryostat wall and L3 lens inside face. Only these metal and glass surfaces remain warm, to reduce the gas load and the possibility of contamination.

The four thermal zones in the cryostat are independently controlled, to protect the optical surfaces during cool-down and warm-up sequences.

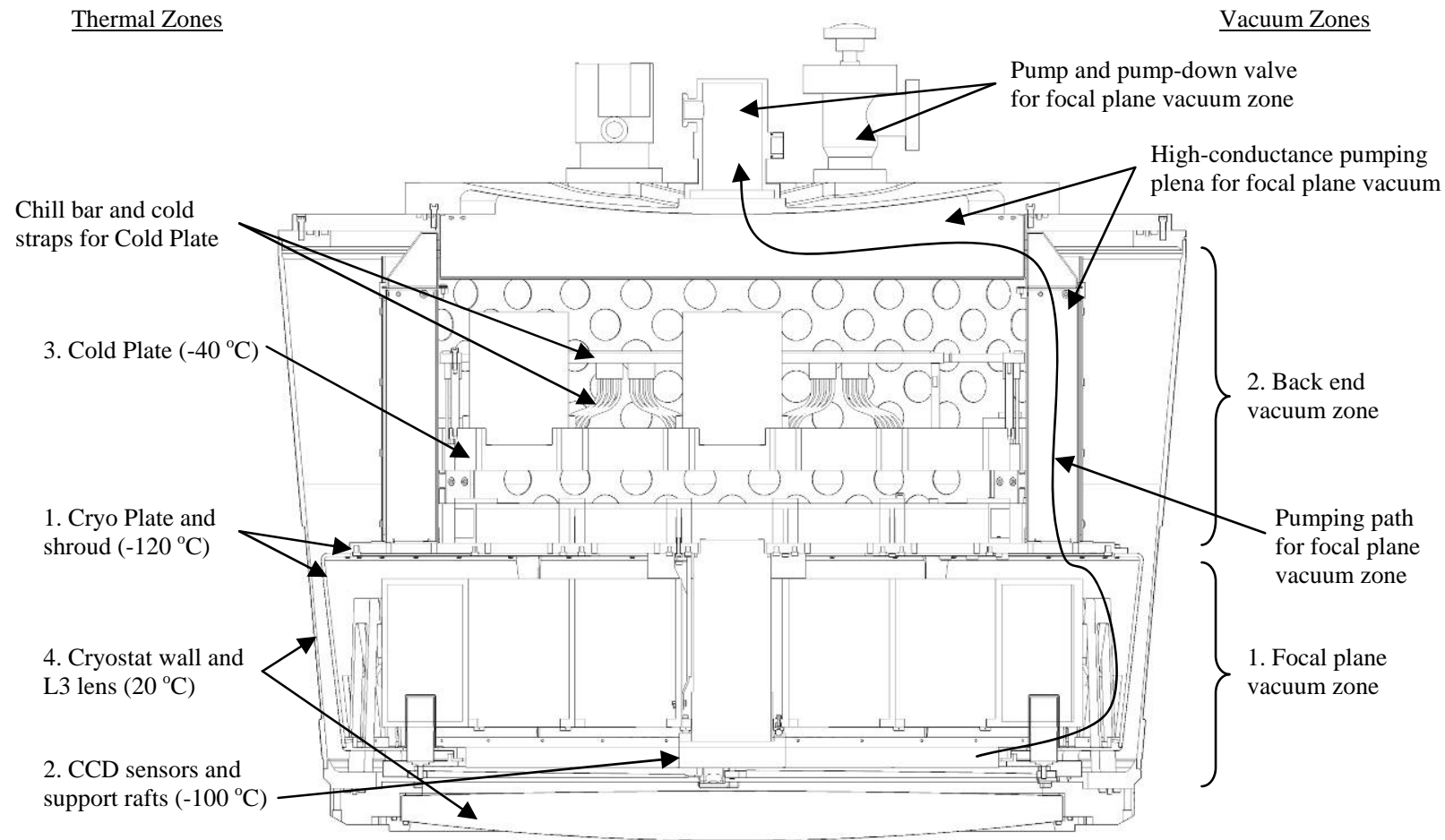

Fig. 4. Schematic of the cryostat thermal zones, annotated on the left, and vacuum zones, annotated on the right; separation of the focal plane by such zoning has helped isolate it from temperature fluctuations and from contamination sources inside the cryostat.

\subsection{Cryostat vacuum zones and pumping}

The cryostat has also been segmented into two vacuum zones. While the thermal zones serve to reduce the rate of outgassing, the separate vacuum zones control the molecular flow of outgassing products within the cryostat, thereby reducing the impact of any gas load on the lens and CCD surfaces.

While the cryostat forms one physical vacuum vessel, it is divided into two vacuum zones which are separated by very low conductance barriers. The focal plane zone includes only the sensor rafts, L3 lens, grid, and front of the cryostat. These are composed largely of metals and ceramics, which will keep both the outgassing and water load to a minimum. The focal plane vacuum zone is pumped separately with dedicated vacuum pumps connected to the focal plane region by high-conductance pumping plena. 
The entire back end of the cryostat forms the second vacuum zone. Nearly all of the electronics components are in this zone, as well as all MLI and most of the warm surfaces. This zone is separately pumped to reduce the possibility of cross-contamination in the pumping manifolds, and is instrumented, to track vacuum levels and gas species.

\subsection{Materials qualification process}

The final element of the contamination control plan is a qualification process for all materials that are planned for use in the cryostat. A materials qualification program is currently underway, to characterize specific requirements for materials in the cryostat, based on the impact of different outgassing products to optical transmission and photometry. The results of these tests will be used for defining which materials are or are not permitted in the cryostat. Ultimately, this materials qualification information will be recorded in a database of allowed materials and processing methods. A good first step in this process is to ensure that all materials are on the NASA materials database (available at: http://outgassing.nasa.gov/), but while this is a necessary initial condition, it is not a sufficient qualifier to ensure opticallevel cleanliness of items in the cryostat. Furthermore, literature searches to-date have shown that there has been little rigorous qualification of materials, suggesting that there is much to be learned about the exact source and nature of fouling of sensors and lenses in a vacuum.

\section{L1/L2 ASSEMBLY}

\subsection{Design overview}

Moving outside the cryostat, the large L1 and L2 lenses are housed in a separate sub-assembly at the front end of the camera. The L1 lens has a clear aperture radius of $775 \mathrm{~mm}$ and forms the window into the camera volume, with a diameter nearly that of the camera itself. With a clear aperture radius of $551 \mathrm{~mm}, \mathrm{~L} 2$ is significantly smaller in diameter, but still large and heavy. As shown in Fig. 5, the two lenses are supported off a toroidal ring with a triangular crosssection. The closed-section ring provides stiffness in a compact package, to stay clear of the lens clear apertures on the inside, while not encroaching on the main light beam from the M2 to M3 mirrors that grazes by on the outside. Each lens is mounted to the ring by way of flexures which isolate the lenses from forces and moments due to distortions of the ring, and ensure that the glass is supported in as benign a way as possible. The entire sub-assembly is itself kinematically supported by a hexapod of adjustable struts, which offset the lenses from the front end of the camera housing. The assembly provides a stiff support for the lenses, while minimizing the strain-induced loads on the glass.
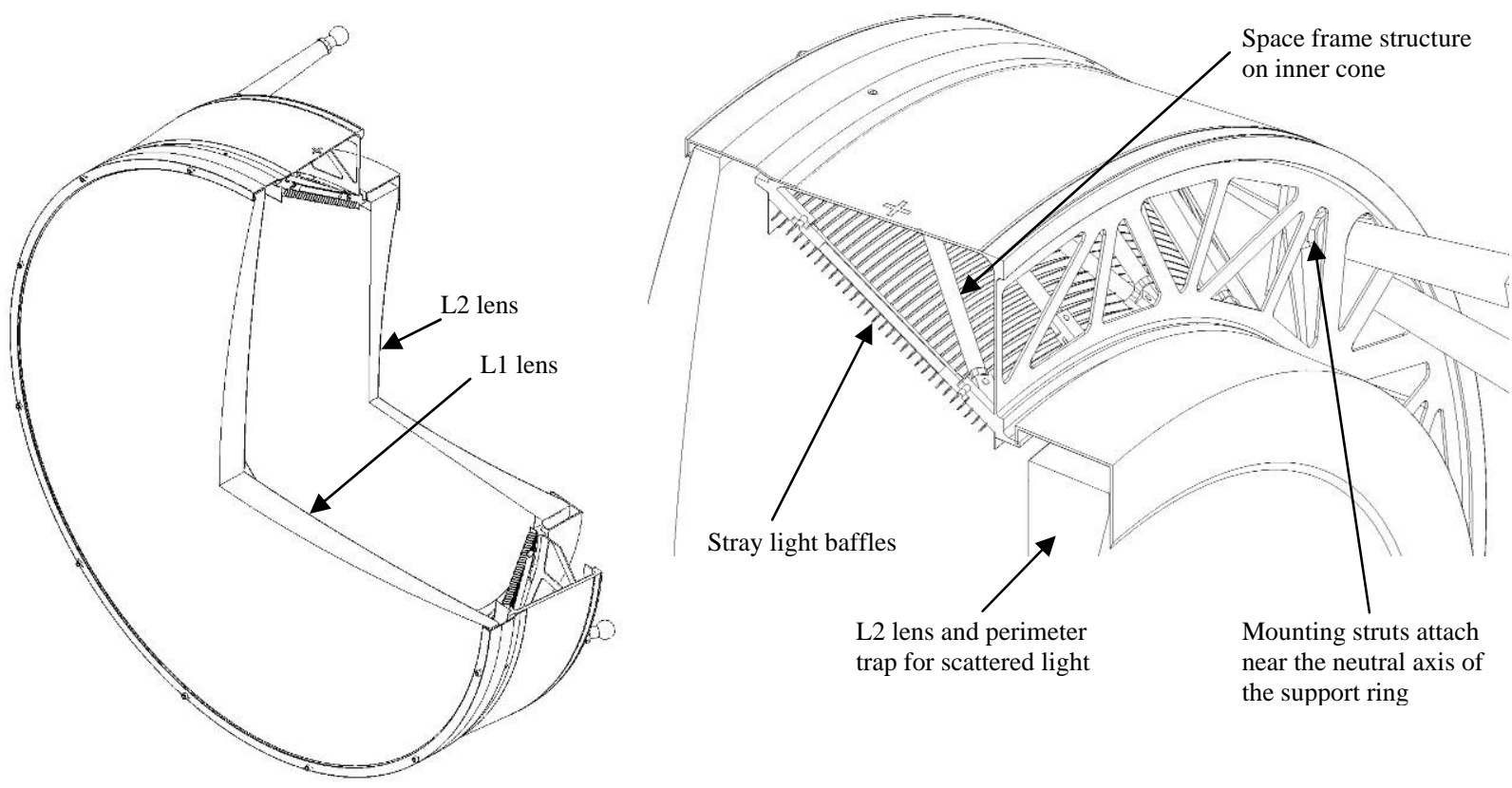

Fig. 5. L1/L2 lens sub-assembly; the annular ring with a triangular cross-section has been structurally optimized to provide adequate stiffness while reducing its weight 


\subsection{Driving requirements}

The L1/L2 sub-assembly design responds to two requirements that have significantly driven the design. These and other requirements and parameters are listed in Table 5. First, the lenses must remain stable with respect to the focal plane while the telescope re-points and the camera rotates about its bore axis. Motion of the lenses translates directly into degradation of image quality, with lens tilt being the most sensitive motion. The camera is supported by a hexapod positioner that can compensate for some of the motions, but this will only zero out the average motion of the two lenses, and does not accommodate changes to deflections as the camera rotates.

In direct opposition to the lens motion requirements is the need to keep the support structure lightweight. The L1/L2 Assembly mounts at the extreme end of the camera, so its mass is offset the farthest from the telescope top end assembly. This results in the largest moments and the biggest impact on the top end natural frequency and response to dynamic loads. Given that the size-thus mass-of the lenses is not negotiable, the mass of the support structure must be carefully optimized to reduce unneeded structural mass while still providing adequate stiffness and stability.

\subsection{Efficient structural design}

The efficient structural design of the L1/L2 assembly responds well to the competing requirements of structural stiffness and lightweight design, by providing an optimal balance of performance. In particular, the closed toroidal shape provides superior stiffness when compared with comparable open section shapes. The structure sees in-plane "squashing" forces from the mass of the lenses, but also undergoes twisting both because the lenses are not aligned with the center of the ring and because the ring tends to warp around its three strut mount points. The total mass of the annular structure is $46.4 \mathrm{~kg}$, which is only $9.7 \%$ of the weight of the lenses and light baffles. This low ratio of structure/payload mass is usually found only in spacecraft structures.

\subsection{Space frame}

The tubular space frame forming the inner cone of the support ring points to the heritage of the design in aerospace structures. Early designs included a sheet metal cone, but structural analysis indicated that the solid cone was not being efficiently loaded, and perforating the cone with lightening holes would produce thin, open-section members that were not well-suited for carrying structural loads, were prone to buckling, and would be difficult to fabricate accurately. However, a space frame of tubular struts provides structural stiffness along with light weight and relatively straightforward manufacturing. The straight struts are axially loaded and are made from very thin wall aluminum tubing with epoxied end fittings. The entire space frame is considerably lighter than the original solid cone, while still providing adequate stiffness to react the twisting loads.

\subsection{Pick-up points}

The lens assembly mounting struts form the final element of the structural design. These support the mass of the entire assembly off the front of the camera, and their mounting locations have been optimized to reduce the impact of the point-loading on the deflection of the lenses. In particular, the struts are actually mounted in the interior of the toroidal ring, near the neutral axis of the triangular cross-section. The shape of the cross-section has been adjusted so that the shear center and center of gravity was brought in line with the neutral axis. This ensured that the cone is supported in a location to minimize deflections from both out-of-plane bending as well as warping from in-plane shear.

The design of the structure supporting the L1 and L2 lenses has been optimized to meet the competing requirements of minimizing lens motion and reducing structural mass; the resulting design performs with an efficiency typical of aerospace structures. 


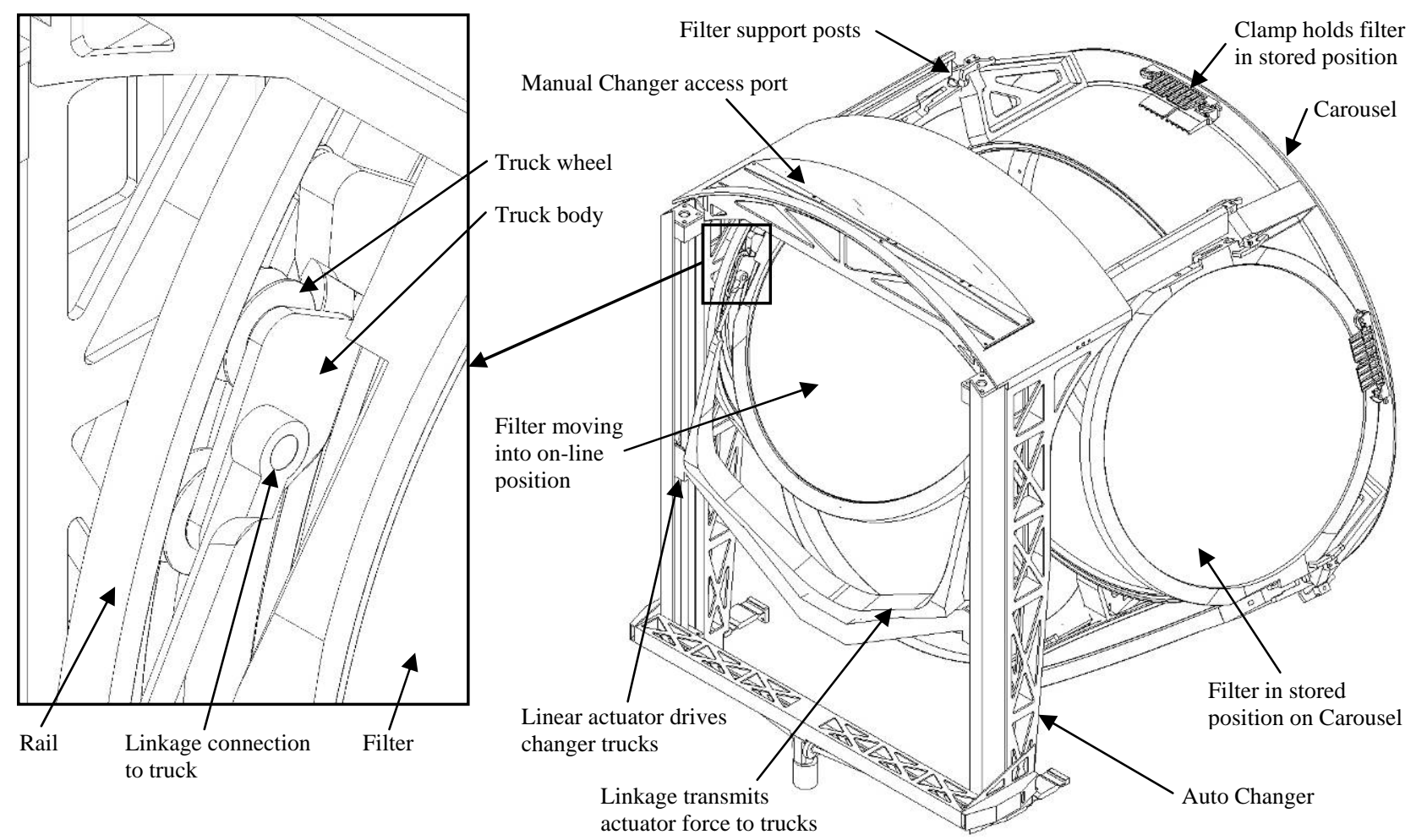

Fig. 6. Filter exchange system with a filter moving into the on-line position; the carousel supports stored filters around the perimeter of the cryostat, while wheeled trucks move on two rails, supporting and guiding the filter as it is moved into position

\section{FILTER EXCHANGE SYSTEM}

\subsection{Design overview}

The LSST camera houses five on-board filters for fast filter changing during nightly viewing, and includes the capability for swapping in a sixth filter during a daytime maintenance accesses. As shown in Fig. 6, the filter exchange system includes three sub-assemblies to provide this functionality. The carousel holds the filters that are not in use, and moves filters into position for placing on-line when needed. The filters sit in cradles on a ring bearing that surrounds the cryostat, with the filters oriented 90 degrees to their on-line position. The selected filter is moved into the light beam by the auto changer. This mechanism grabs the filter while it sits in its slot in the carousel, then moves it forward and pivots it into its final position between the L2 and L3 lenses. Finally, a manual changer is used for swapping out a filter during daytime access. The manual changer engages with the internal auto changer rails to ensure a smooth and safe hand-off of the filter. All three of these assemblies ensure that the large-diameter filters are handled safely, and can be accessed and brought on-line quickly to maximize viewing time.

\subsection{Driving requirements}

The mechanical design of the filter exchange assemblies has been largely driven by three requirements. These and other requirements are listed in Table 6. First, the primary derived requirement for the system is that any of five filters can be inserted into the on-line position in less than 120 seconds. This relatively fast change-out time is one of a number of important parameters to maximize the cadence of image-taking during nightly viewing, which is essential for a survey telescope such as LSST. There will be 10-15 filter changes per night, so just the act of changing

Table 6. Filter exchange system key requirements and parameters

\begin{tabular}{|l|l|}
\hline \multicolumn{1}{|c|}{ Property } & \multicolumn{1}{c|}{ Value } \\
\hline Filter clear aperture diameter & $690 \mathrm{~mm}$ \\
\hline Max filter exchange time & $120 \mathrm{sec}$ \\
\hline Max filter mass & $35.5 \mathrm{~kg}$ \\
\hline Number of on-board filters & 5 \\
\hline
\end{tabular}


filters will use about $5 \%$ of the nightly viewing time. Second, the large size of the focal plane and filter clear aperture significantly impacts all aspects of the design of the filter exchange system. Finally, along with handling the large filters, the exchange system must fit within the envelope of the camera and clear the bore of the M2 mirror. The filter clear aperture is a large fraction of the overall camera diameter, so sliding-filter or cartridge-changer designs simply do not fit.

\subsection{Carousel filter storage}

To meet the tight constraints on physical packaging constraints of the filters, the exchange system shuttles filters from their storage carousel, forward and around the edge of the cryostat and L3 lens, to the on-line position between the L2 and L3 lenses. This system includes handing off the filter from the carousel storage mechanism to the auto changer. While simple one degree-of-freedom linear rail systems may work for cameras with smaller focal planes, such planar systems use far too large a footprint to fit into the constrained volume of the LSST camera. This has been the primary driver in designing a more complex three degree-of-freedom system. This system includes the carousel which holds up to five filters and rotates around the camera's bore axis to place the selected filter in the hand-off position. The filters are arrayed around the bore axis in a pentagonal configuration, facing radially inward towards the center of the camera. The carousel includes fail-safe clamps to hold the filters in place while they are in this off-line position.

\subsection{Auto changer}

The auto changer mechanism provides the second and third actuated degrees of freedom to move a selected filter from its parked position in the carousel forward and around L3 into the on-line position. To do this, the changer must translate the filter along a curved path as well as rotate it 90 degrees. Nine types of mechanisms were investigated which could produce this composite motion, including four-bar linkages, gantries, articulating "robot" arms, and various rail systems.

Ultimately, a so-called "double-rail" system was chosen. A filter is carried by a truck that rides along a set of rails which moves it into position. A second, nested rail guides an auxiliary wheel that pivots the filter. Changing the offset between the two rails alters the pivot angle of the filter. Thus, by using only one actuator to drive the trucks along their rails, the filter is moved into position while undergoing a coordinated rotation, clearing neighboring obstacles. The path of the rails in the double-rail mechanism can be designed to produce nearly any filter path necessary. In particular, the path of the filter must clear the edge of the shutter frame, while still not colliding with the L2 lens on the leading edge or the outer housing of the camera at the trailing edge. This design flexibility has proven invaluable in modifying the path of travel to clear obstacles as component designs change.

The double-rail auto changer design, coupled with the storage carousel, has proven to be the only viable option of the many investigated. This provides a robust solution to an otherwise-intractable packaging problem, while providing the fast filter exchange time and long-lived design needed for operations in a survey camera.

\section{SHUTTER}

\subsection{Design overview}

The shutter for the LSST camera lies tightly packaged between the L3 lens/cryostat window, and the filter. It controls the length of time that the sensors on the focal plane are exposed to an image while blocking out all stray light when closed during read-out of the CCD sensors. As shown in Fig. 7, the shutter is a double-acting guillotine-like device, where flat plates move across the focal plane to start or end an exposure. However, because of the tight lateral space constraints, the one-piece shutter has been segmented into three flat blades which are stacked up when the shutter is open, then slid over the field of view in a coordinated accordion-like motion to close.

\subsection{Driving requirements}

The primary performance requirement driving the mechanical design of the shutter is the need for tight control of exposure time across the entire field of view. Collectively, the exposure-control requirements have a direct bearing on the technology choices and implementation of the shutter design, and as a result drive the design of the shutter. Three of the requirements in Table 7 address this: minimum exposure time, time stamp or opening speed, and exposure time uniformity. Meeting these requirements is essential to reducing errors in focal plane exposure, and thereby achieving the performance requirement of the survey. 
The design implementation is also significantly affected by the very tight space constraints for the shutter, both along the bore axis between L3 and the filters, and transversely inside the limited-diameter camera housing. Finally, technology choices are limited to those that can meet the long cycle lifetime without adverse contamination from wear or lubrication.

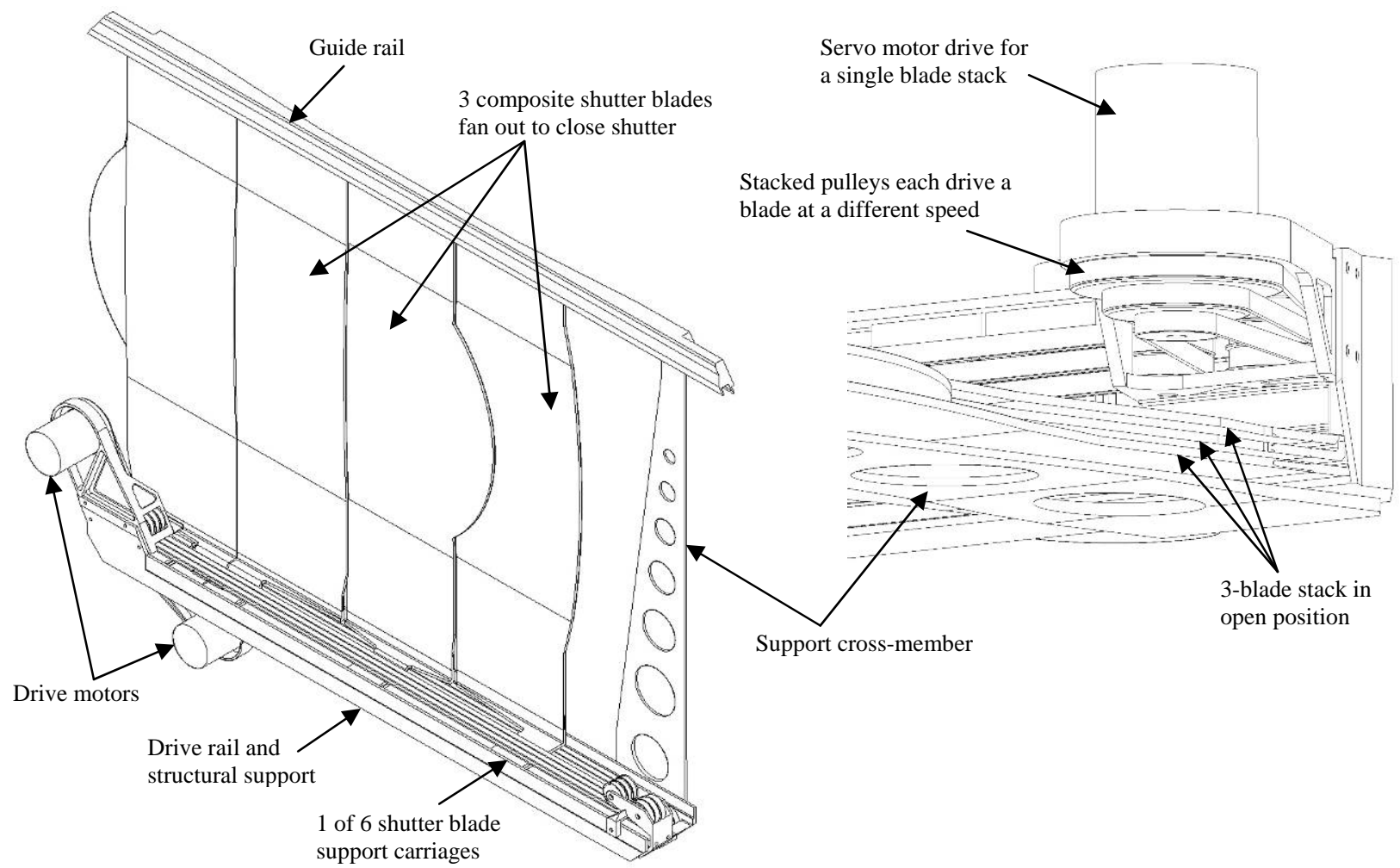

Fig. 7. Shutter in the closed position, showing one set of three blades spread out over the field of view; the second set of three blades is stacked up on the left side of the shutter; the detail to the right shows one set of pulleys and timing belts, driven by a single motor but with different diameters to drive blades at different speeds

\subsection{Shutter design details}

The double-acting shutter design follows directly from the requirements for tight control of exposure time. A singleacting shutter simply retracts to one side to clear the field of view (FOV) during the exposure, then re-traces its path to close. However, such an out-and-back design cannot provide the needed exposure time uniformity. Alternately, a double-acting shutter essentially includes two shutters. The first moves off to one side to clear the FOV to start the exposure, then the second shutter occludes the FOV by coming on from the opposite side, and traveling in the same direction as the first. For the next exposure, the process is reversed. The advantage of the double-acting design is that it provides a more uniform exposure time for all pixels. The first pixel exposed by the trailing edge of the first shutter is also the first blocked by the leading edge of the second. Furthermore, the double-acting, straightedged shutter design allows close control of the exposure time for each pixel, by ensuring that both leading and trailing edges of the shutter blades follow the same acceleration and velocity profiles.

While double-guillotine shutter designs have been used on a number of cameras, tight space constraints have led to the novel stacked-blade design for the LSST shutter. Three stacked blades form one shutter, and move across the focal plane in unison as the shutter opens. The three blades are formed, three-dimensional shapes, manufactured from carbon-fiber face sheets and a high-density foam core. The blades are supported at both ends,

Table 7. Shutter key requirements and parameters

\begin{tabular}{|l|l|}
\hline \multicolumn{1}{|c|}{ Property } & \multicolumn{1}{c|}{ Value } \\
\hline Minimum exposure time & $1 \mathrm{sec}$ \\
\hline Opening/closing time & $0.5 \mathrm{sec}$ \\
\hline Exposure time uniformity & $100 \mathrm{~m}-\mathrm{sec}$ \\
\hline Max shutter thickness & $19.1 \mathrm{~mm}$ \\
\hline Cyclic lifetime & $10^{7}$ cycles \\
\hline
\end{tabular}


but only driven from one end, where they each are supported by separate carriages on a single rail. The carriages are attached to a single drive motor by way of separate timing belts that wrap around timing pulleys of differing diameters, moving the three blades in unison but at different speeds. Thus, a single motor drives all three blades to extend and retract over the entire FOV.

Likewise the opposing stack of three blades shares the same rail—six carriages on a single rail—and includes it own drive motor and pulley set. A single rail is used for all six blades, since only one set of blades covers the field of view at a time. This design is elegant and efficient, using the minimum of components to drive the blades, while ensuring that the timing requirements are met by implementing proven timing-belt technology that has been used in the automation industry for many years.

\section{DESIGN FOR INTEGRATION AND TEST}

\subsection{Modularity}

Three overarching design principals have guided the camera design as it has evolved and been refined,. First, camera components should be modular and self-contained. This allows for independent assembly of components and assemblies at remote locations within the collaboration, as well as early in-process testing of the modules before they are integrated in the camera. This both reduces the risk of problems during camera integration, and speeds the final integration process. Component modularity also improves serviceability by reducing the mechanical interdependencies between components, and allowing them to be serviced and removed with minimal disturbance of neighboring components.

\subsection{Verification}

Second, only pre-tested and verified sub-assemblies and components are integrated into the camera assembly. All key camera sub-assemblies will be both functionally and performance tested prior to installing in the camera, thereby minimizing the risk of early failures and the need for disassembly during follow-on integration work and early commissioning. Furthermore, performance is again verified after integration in the camera, but before subsequent work is performed. Such methodical incremental testing ensures that the outcome of each integration step is verified to be successful, and that any process or procedural problems are caught early.

\subsection{Planning for integration and servicing}

Third, early planning for integration and testing—and the related servicing activities during operations—is essential during the development phase, and provides a strong base for implementing the design and starting construction. The camera mechanical package is heavily constrained by its optical and structural requirements, and the control systems require tight inter-relationships among subsystems. Thus, planning for and understanding how these are brought together and tested is required to ensure that they operate as expected when they have been fully assembled.

\section{CONCLUSION}

The structural and thermal design of the LSST camera reflects the driving performance requirements of system stability in a small package. This has been accomplished by isolating key optical, structural, and thermal elements while also developing efficient systems that maximize mechanical performance within the tight space constraints of the telescope. Furthermore, modular component designs have been implemented to leverage the organization of the project, while ensuring that systems are fully verified and are easily serviceable during observing. The camera mechanical design and implementation plans meet the operation needs and performance requirements of this next-generation survey telescope.

\section{ACKNOWLEDGEMENTS}

LSST is a public-private partnership. Funding for design and development activity comes from the National Science Foundation, private donations, grants to universities, and in-kind support at Department of Energy laboratories and other LSSTC Institutional Members. Work supported by the Department of Energy contract DE-AC02-76SF00515. 